\title{
Dark matter searches using superheated liquids
}

\author{
Bou-Cabo Manuel ${ }^{2, a}$, Ardid Miguel ${ }^{1}$, and Felis Ivan $^{1}$ on behalf of PICO Collaboration \\ ${ }^{1}$ IUPV-IGIC, Universidad Politécnica de Valencia, Spain \\ ${ }^{2}$ IFIC, Universidad de Valencia, Spain
}

\begin{abstract}
Direct detection of dark matter is one of the most important topics in modern physics. It is estimated that $22 \%$ of universe matter is composed by dark matter in front of $0.4 \%$ of ordinary matter like stars, galaxies planets and all kind of known astrophysical objects. Several kinds of experiments are nowadays involved in detection of one of the more accepted particle candidates to be dark matter: WIMPs (Weakly Interacting Massive Particles). These detectors, using several kinds of techniques: Cryogenic semiconductors, scintillation materials like I Na or noble gas chambers among others, are reporting very interesting but inconclusive results. In this paper a review of detectors that are using the superheated liquid technique in bubble chambers in order to detect WIMPs is reported. Basically, we will report about Coupp (Chicagoland observatory for underground particle physics), PICO that is composed by Coupp and Picasso researchers having the aim to build a ton experiment and also about a new detector named MOSCAB (Materia oscura a bolle) that recently published a first results of a test chamber that uses also superheated liquid technique but as a Geyser chamber.
\end{abstract}

\section{Introduction}

Direct detection of WIMPs is commonly accepted like the most used way to try to detect non baryonic dark matter of the Universe. This paper is related with the use of superheated liquid to detect nuclear recoil produced by WIMP interaction with nucleus present in target material. The most tested and succesful detector of dark matter that uses this technique is the COUPP/PICO bubble chamber detector. Basically, it consists of a bubble chamber filled with $\mathrm{CF}_{3} \mathrm{I}$ or $\mathrm{C}_{3} \mathrm{~F}_{8}$ that operates in superheated regime. Detection of nuclear recoil is based on bubble formation. When nuclear recoil is produced with enough energy and this energy is deposed in the medium in a space smaller than the critical radius for a given thermodynamic conditions a macroscopic bubble is generated. Several authors have described the growth of the bubble in a superheated liquid, the first ones were Rayleigh [1], Plesset \& Zwick [2] or Foster and Zuber [3] that arrive to approximate solutions of bubble growth rate, ((1) Rayleigh - Plesset equation).

$$
\frac{\mathrm{p}_{\mathrm{v}}\left(\mathrm{T}_{\mathrm{v}}\right)-\mathrm{P}_{\infty}}{\rho_{l}}=\mathrm{R} \cdot \frac{\mathrm{d}^{2} \mathrm{R}}{\mathrm{d}^{2} \mathrm{t}}+\frac{3}{2} \cdot\left(\frac{\mathrm{dR}}{\mathrm{dt}}\right)^{2}+\frac{4 \cdot \eta_{1}}{\mathrm{R}} \cdot \frac{\mathrm{dR}}{\mathrm{dt}}+\frac{2 \cdot \sigma}{\rho_{1} \cdot \mathrm{R}}
$$

being $\mathrm{p}_{\mathrm{v}}\left(\mathrm{T}_{\mathrm{v}}\right)$ pressure within the bubble, $\mathrm{P}_{\infty}$ external pressure far away from the bubble, $\eta_{1}$ represents the kinematic viscosity of the fluid, $\sigma$ surface tension of the bubble, $\rho_{1}$ density of fluid and $\mathrm{R}(\mathrm{t})$ is the radius of the bubble.

\footnotetext{
a e-mail: manuelbcabo@gmail.com
} 
In the case that is being treated here, the liquid is in a superheated state that basically consists in having the liquid to a pressure such that is kept in liquid state at a temperature above its evaporation temperature.

The growth of the bubble is composed by three different processes attending to the nature of the bubble formation:

- Nucleation

- Regime dominated by inertia where (radius $\alpha$ time)

- Regime dominated by heat diffusion where (radius $\alpha$ time ${ }^{1 / 2}$ ).

In order to determine the thermodynamic conditions of the superheated liquid in terms of pressure and temperature of the fluid that fix the desired energy threshold of the detector for nuclear recoils, the "hot spike" Seitz model [4] is applied. This model considers the necessary energy to overcome the surface energy and the latent heat, in first approximation the energy threshold can be parameterized as follows:

$$
\mathrm{E}_{\mathrm{th}}=4 \cdot \pi \cdot \mathrm{r}_{\mathrm{c}}^{2} \cdot\left(\sigma-\mathrm{T} \cdot \frac{\delta \sigma}{\delta \mathrm{T}}\right)+\frac{4}{3} \cdot \pi \cdot \mathrm{r}_{\mathrm{c}}^{3} \cdot \rho_{\mathrm{v}} \cdot\left(\mathrm{h}_{\mathrm{b}}-\mathrm{h}_{1}\right)
$$

where $\sigma$ is the surface tension, $\mathrm{T}$ is the temperature of the fluid, $\rho_{\mathrm{v}}$ is the bubble vapour density, $h_{1}, h_{b}$ are the specific enthalpies of the bubble vapor and superheated fluid and $r_{c}$ represents the critical radius of the bubble.

Detection of WIMP nuclear recoils using superheated liquid technique presents important advantages with respect other techniques, such as strong rejection of minimum ionizing particles and gamma rays or electron background coming from gamma rays or beta decay, easy and cheap mechanical construction, possibility of use different target material being possible distinguish among spin dependent and spin independent interactions and measuring in a simple way multiple neutron interactions that can help to avoid later this kind of background. In next sections the operation mode of this kind detectors (Sect. 2) will be discribed and results obtained by COUPP $[5,6]$ collaboration will be discussed.The future plans for new merged PICO (Picasso [7] + COUPP) collaboration will be presented as well as the new european initiative, MOSCAB [8], to build a detector able to detect dark matter via WIMP nuclear recoil using superheated liquid using the "geyser" technique. Finally the conclusions of this communication will be summarized in Sect. 4.

\section{Detectors and collaborations}

The basic description of the bubble chamber detectors for dark matter detection consists of a fused silica quartz vessel immersed in a hydraulic fluid (propylene glycol commonly used), a pressure control unit to maintain the pressure inside and outside of the jar at a desired value to put the target material in a superheated state. All this equipment is contained inside of stainless steel pressure vessel. The inner part of the bubble jar is recorded by 2 VGA cameras with a typical sample frequency of $\sim 100$ frames/second allowing to reconstruct the spatial coordinates of bubbles inside of the vessel. The temperature is also monitored using temperature sensors mounted along the detector with the aim to control the thermodynamic conditions of target material. An important part of the instrumentation used is composed by several piezo transducers attached to the wall of the vessel. These piezo transducers can be used to register the acoustic signal produced by the bubble growth when nuclear recoil is produced. As it will be shown at next section the acoustic signal emitted by the bubble growth gives us the possibility to distinguish between neutron nuclear recoils, and alpha nuclear recoils, being very useful in terms of alpha background rejection. 
Moving specifically to COUPP/PICO detectors, the superheated state of target material is achieved performing cycles of compression and decompression of the chamber. Typically the chamber is in a compressed mode at a pressure of $\sim 215$ psia, the superheated state is achieved performing a decompression going from 215 psia to tens of psia (depending of the energy threshold chosen) and after a stabilization time the detector is able to "look and listen" wimp interaction for a period of hundreds of seconds (typically around $500 \mathrm{~s}$ ). During this time an algorithm compares consecutive frames and if some pixels change the trigger of the detector is activated and the event detected is recorded, typically $50 \mathrm{~ms}-100 \mathrm{~ms}$ of signals are recorded. After that, the bubble chamber is compressed and the cycle starts again.

The main difference between COUPP/PICO and MOSCAB detectors is that with the use of "geyser" technique, MOSCAB operates by controlling the temperatures of the superheated liquid and the colder vapour and therefore does not have cycles of compression and expansion. Once the bubble is created at MOSCAB detector it goes up passing through a gradient of temperature that collapses the bubble from vapour to liquid. This has the advantage of not needing to reset the bubble chamber, decreasing the dead time of the detector. During last 5 years, the COUPP collaboration developed few detectors of different size, performing several physics runs running at different levels of energy threshold.

One common system present in this kind of detectors is the acoustic system that plays an important role in background rejection. The major part of background rejection can be avoided considering the location of the detectors placed at deep underground laboratories like SNOLAB for COUPP/PICO detectors, but it is necessary to have an additional method to reject the alpha background. This rejection [9] has been achieved obtaining using the acoustic discrimination based on the calculation of an acoustic parameter that basically calculates the power spectrum density of the acoustic signal generated by the bubble growth. In COUPP/PICO detectors the acoustic parameter is obtained using Eq. (3).

$$
\mathrm{AP}=A(\mathrm{~T}) \cdot \sum_{\mathrm{j}} \mathrm{G}_{\mathrm{j}} \cdot \sum_{\mathrm{n}} \mathrm{C}_{\mathrm{n}}(\overrightarrow{\mathrm{x}}) \cdot \sum_{\mathrm{f}_{\min }^{\mathrm{n}}}^{\mathrm{f}_{\max }^{\mathrm{n}}} \mathrm{f} \cdot \mathrm{psd}_{\mathrm{f}}^{\mathrm{j}}
$$

being $A(T)$ a temperature dependent scale factor, $G_{j}$ the gain of the acoustic transducer $j$, $C_{n}(\vec{x})$ a position correction factor, $f$ is the frequency and $\operatorname{psd}_{f}^{j}$ represents the power spectrum density centered at frequency bin $f$ for transducer $\mathrm{j}$. In next section the results obtained for alpha rejection in COUPP/PICO family detectors will be shown.

The schedule of COUPP \& PICO family detectors can be summarized as follows.

\section{COUPP-4:}

It was a 2-Litre bubble chamber filled with $\mathrm{CF}_{3} \mathrm{I}$. This detector was running at Snolab from 2010 to 2012, being stopped to implement and install some improvements in its instrumentation. Some of this changes involved the replacement of acoustic transducers, due to find some contamination at PZ 27 piezo electric ceramic, specifically were found $4 \mathrm{ppm}$ of $U$ and $2 \mathrm{ppm}$ Th. New generation of piezo transducers, were developed using radio clean piezo ceramic salt. The new piezos were used during the second physics run.

\section{COUPP-60:}

Second detector implementing the superheated liquid technique was developed by the COUPP collaboration after the results of the first $4 \mathrm{~kg}$ bubble chamber built. The aim of this detector was increasing the effective volume of the target material filling the vessel up to 40 litres of $\mathrm{CF}_{3} \mathrm{I}$. In spite of all implemented systems works fine, some problems were found due to the appearance of some 'anomalous events' at the neutron recoil zone. 


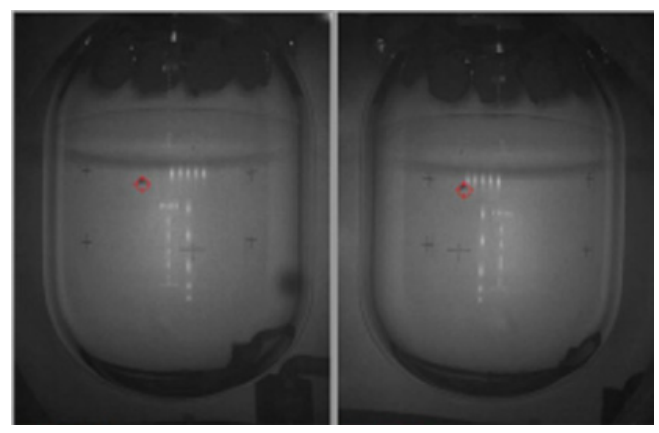

Figure 1. Detail of single bubble event at Coupp-4.

It is planned for 2015 to upgrade the system to solve this problem installing a microfiltering system to avoid micro impurities that could be the cause of the problem. Also the target material could be changed in order to use $\mathrm{C}_{3} \mathrm{~F}_{8}$.

\section{PICO-2L:}

Since 2013 PICASO and COUPP collaborations merged in a new one named PICO. The PICO - $2 \mathrm{~L}$ detector is the first detector built by the new collaboration. This $2 \mathrm{~L}$ bubble chamber has been taken data during 2014 and the main difference with respect to the previous commented detectors, is the change of the target material, using $1 \mathrm{C}_{3} \mathrm{~F}_{8}$ that has twice fluorine density in comparison with $\mathrm{CF}_{3} \mathrm{I}$ and a lower energy threshold can be achieved. After almost a year of data taking and observing arather good behaviour of all systems integrated at the detector, the detector has been stopped and probably a new physics run will be performed maybe using $\mathrm{CF}_{3} \mathrm{I}$.

\section{PICO-250L:}

The mean objective for PICO collaboration consists of building a ton scale detector based on 250L bubble chambers. The plan is starting to build the chamber in 2016, but the final timing will depend on the results obtained from previous COUPP/PICO detectors.

\section{MOSCAB:}

MOSCAB stands for (Materia Oscura A Bolle) and represents an european initiative promoted by U. Milano-Biccoca team leaded by professor A. Pullia, with the aim to build a bubble chamber able to detect wimp nuclear recoil. A first prototype has been tested using $0.5 \mathrm{~kg}$ of $\mathrm{C}_{3} \mathrm{~F}_{8}$, at U. Milano-Biccoca laboratory. A $40 \mathrm{~kg}$ detector is now being commissioned and the future plans consider moving the detector to Gran Sasso laboratory [10].

The main results obtained for each detector are discussed in next section, giving an idea of the state of art of superheated liquid chamber detectors for dark matter searches.

\section{Results}

In 2010 begun to take data de first COUPP detector, that was bubble chamber filled with 2 liters of CF3I. An extensive information about results obtained can be found at [5], but can be summarized as follows.

The total exposure of the detector was $553 \mathrm{~kg}$-day and were performed 3 levels of energy threshold. Multibuble events were recorded, but the counts observed were in good agreement 


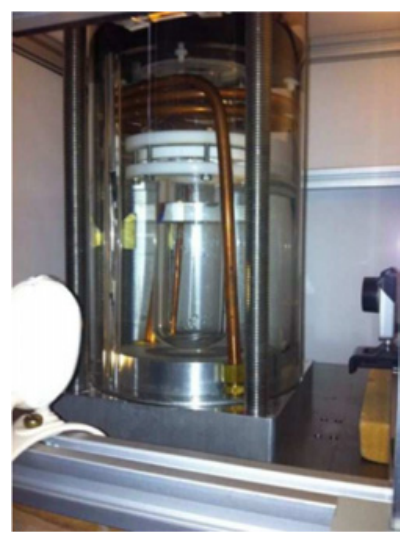

Figure 2. MOSCAB detector installed on Milano - Biccoca laboratory.

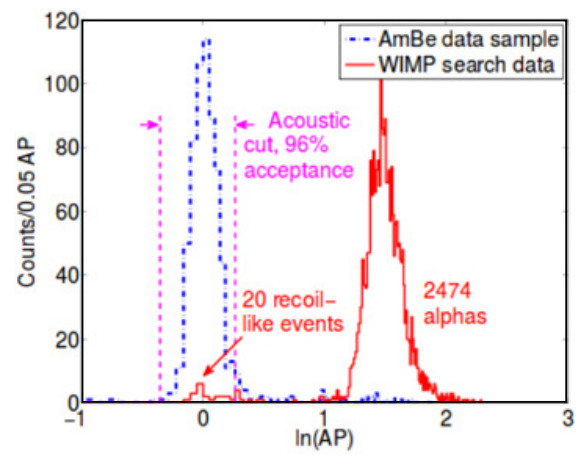

Figure 3. Distribution of acoustic parameter for $553 \mathrm{~kg}$-day compilation data. The alpha background rejection presents a $96 \%$ of acceptance for neutron recoil zone. In this physics run considered 20 like WIMPs events were detected at the zone of neutron nuclear recoil.

with the total neutron background expected. The total exposure for single bubble events was $437 \mathrm{~kg}$-day. Concerning about the alpha background rejection using acoustic techniques, results obtained were very good in terms of acoustic neutron acceptance $\sim 96 \%$ and alpha background rejection $\sim 99 \%$ as can be observed at Fig. 3 .

A total of 20 like WIMP events were observed: 6 measured at 7,8 keV threshold level, 6 at $11 \mathrm{keV}$ and 8 at $15,5 \mathrm{keV}$. Acoustic discrimination technique has been revealed a powerful alpha rejection method.

After obtaining these results, the next detector developed was COUPP-60. This detector has accumulated over $4,500 \mathrm{~kg}$-day of exposure running $80 \%-95 \%$ of live time at $11 \mathrm{keV}$ threshold level. All systems that compose the detector worked properly during this time, and no major problems were observed during this time from technical point of view. As it can be observed in Fig. 4 the neutron AP distribution of calibration events presents a narrow shape and good defined region for neutron nuclear recoil events.

However, during the analysis of experimental data anomalous events overlapped with neutron calibration zone were found (Fig. 5). The nature of these anomalous events remains unclear yet but some hypotheses are under discussion, being considered the presence of micro impurities in the $\mathrm{CF}_{3} \mathrm{I}$ the most probable cause. 


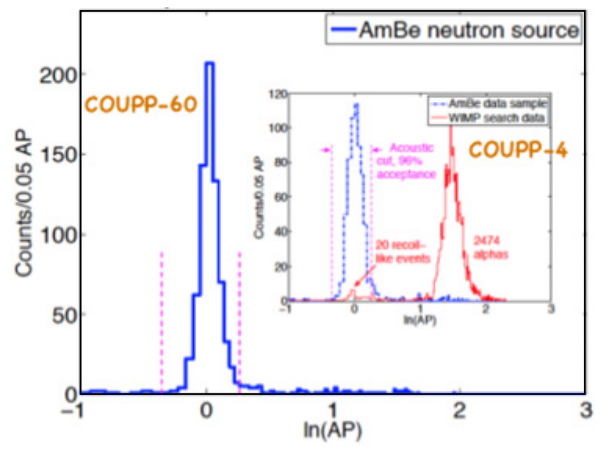

Figure 4. Acoustic parameter distribution for neutron recoil like WIMPs events (COUPP60).

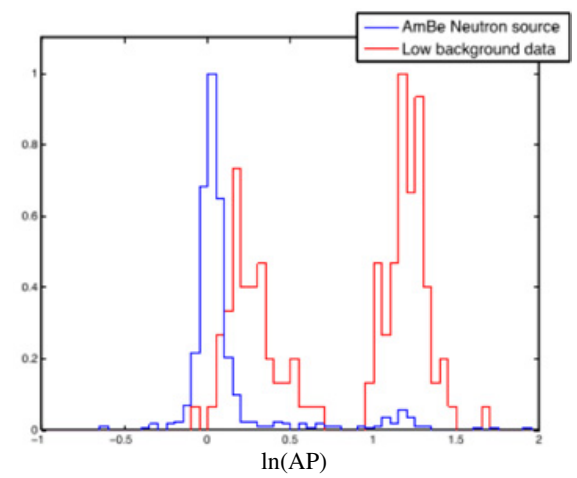

Figure 5. Overlap found in COUPP60 detector, probably due to the presence of micro impurities in the target material.

Hopefully, this data can still be used in spite of the presence of the anomalous events, going further in the acoustic analysis implementing new parameters that can discriminate as much as possible among the two kinds of events. For the near future, it is planned to install a micro-filtering system with the aim to mitigate this problem. The first detector built under the PICO collaboration is a bubble chamber of 2 liters with $\mathrm{C}_{3} \mathrm{~F}_{8}$. The use of this new target material has the advantage to have twice density of fluorine with respect to previous target and being also possible to decrease the energy threshold of the detector. It is important to comment that during the physics run performed by this detector a level of $3 \mathrm{keV}$ was successfully achieved. The acoustic techniques in this case have been also applied successfully and the presence of anomalous events was much more smaller, obtaining a good separation between alpha background and neutron event zone. Figure 6 presents preliminary results for this detector in relation with alpha background rejection and defined zone for WIMP-like nuclear recoil events.

With respect to the results obtained with PICO-2L, the final analysis is almost complete and soon they will be published. A second physics run is scheduled to be performed during next months.

Attending to results obtained for all COUPP/PICO family detectors, the limits (and sensitivity projections for the future PICO 250L) for spin dependent and spin independent interactions are shown in Fig. 7 and compared with other experiments. 


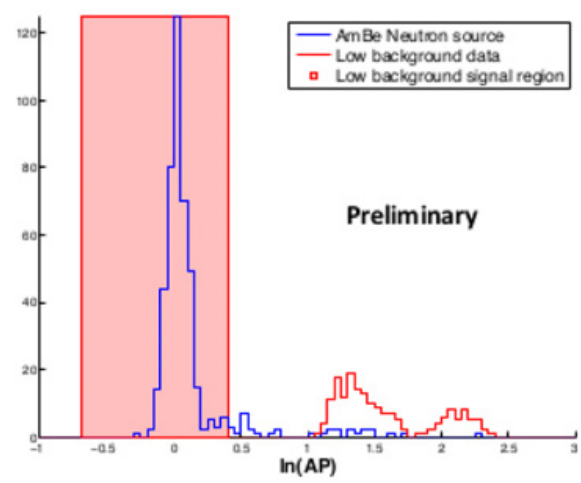

Figure 6. Acoustic parameter distribution for neutron recoil like WIMPs events (PICO2L).
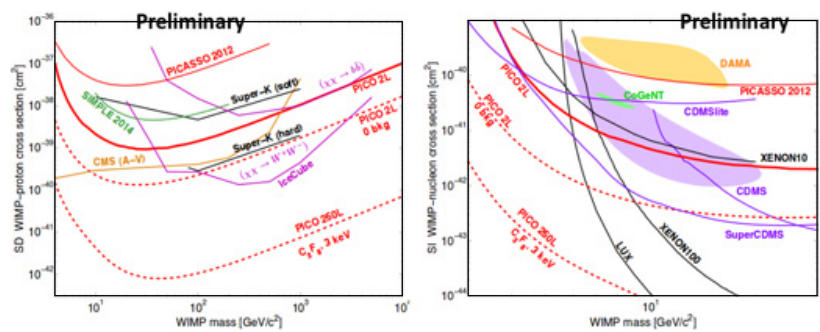

Figure 7. Limit projections for Pico 250 for Spin dependent and Spin independent interaction. Also is included in this plot preliminary results obtained for Pico $2 \mathrm{~L}$ assuming data collected during its first physics run and also assuming 0 background.

With respect to the results obtained with PICO-2L, the final analysis is almost complete and soon they will be published. A second physics run is scheduled to be performed during next months. Attending to results obtained for all COUPP/PICO family detectors, the limits (and sensitivity projections for the future PICO 250L) for spin dependent and spin independent interactions are shown in Fig. 6 and compared with other experiments.

\section{Conclusions}

Summarizing all results obtained by detectors presented previously it seems clear that this kind of detectors will play an important role in direct dark matter detection. Following results obtained for PICO-2L detector the best world limit for proton-WIMP spin-dependent interaction has been achieved.

With respect to results for COUPP-60 bubble chamber, analysis of data is still ongoing trying to minimize the impact of anomalous events on physics run data. In any case, a new physics run with upgraded equipment is ongoing, so future cleaner data will be taken soon.

In addition, a new collaboration with the aim to build a dark matter detector using superheated liquids in a geyser detector has been appeared, obtaining in the first tests very good results in terms of functionality of all systems that compose the detector. News about the installation and tests of the detector in Gran Sasso facilities will come soon. 


\section{References}

[1] L. Rayleigh, On the pressure developed in a liquid during the collapse of a spherical cavity (Philos. Mag 34, 94, 1917)

[2] M.S. Plesset, The growth of vapor bubbles in superheated liquid (J. Appl. Phys. 25, 493, 1954)

[3] H.K. Forster, Growth of vapor bubbles in superheated liquid (J. Appl. Phys. 05, 474, 1954)

[4] F. Seitz (Phys. Fluids 1, 2, 1958)

[5] E. Behnke et al., Coupp Collaboration, First dark matter search results from a 4-kg CF3I bubble chamber operated in a deep underground site (Phys. Rev. D 86, 2012)

[6] Behnke et al., Coupp Collaboration, Direct measurement of the bubble-nucleation energy threshold in a CF3I bubble chamber (Phys. Rev. D 88, 2013)

[7] S. Archambault, Picasso Collaboration, Dark Matter Spin-Dependent Limits for WIMPs Interactions on ${ }^{19} \mathrm{~F}$ by PICASSO (Phys. Lett. B, 2009)

[8] R. Bertoni et al., A new technique for direct investigation of dark matter (Nuclear Instruments and Methods in Physics Research, A, 61-68, 2014)

[9] M.Bou-Cabo et al., LOW Radioactivity Techniques 2013 (LRT 2013): Proceedings of the IV International Workshop in Low Radioactivity Techniques, V: 1549, 142 - 147

[10] M. Ardid et al., MOSCAB: Direct dark matter search using the Geyser technique, Proc. ICHEP 2014 Conf., Nucl. Phys. B: Proc. Supp. (in press) 